\title{
RESEARCH
}

\section{Proof of the Bessenrodt-Ono Inequality by Induction}

\author{
Bernhard Heim ${ }^{1,2^{*}}$ (10) and Markus Neuhauser ${ }^{2}$
}

\author{
*Correspondence: \\ bernhard.heim@rwth-aachen.de \\ 'Lehrstuhl A für Mathematik, \\ RWTH Aachen University, 52056 \\ Aachen, Germany \\ Full list of author information is \\ available at the end of the article
}

\begin{abstract}
In 2016 Bessenrodt-Ono discovered an inequality addressing additive and multiplicative properties of the partition function. Generalizations by several authors have been given; on partitions with rank in a given residue class by Hou-Jagadeesan and Males, on k-regular partitions by Beckwith-Bessenrodt, on k-colored partitions by Chern-Fu-Tang, and Heim-Neuhauser on their polynomization, and Dawsey-Masri on the Andrews spt-function. The proofs depend on non-trivial asymptotic formulas related to the circle method on one side, or a sophisticated combinatorial proof invented by Alanazi-Gagola-Munagi. We offer in this paper a new proof of the Bessenrodt-Ono inequality, which is built on a well-known recursion formula for partition numbers. We extend the proof to the result by Chern-Fu-Tang and its polynomization. Finally, we also obtain a new result.

Keywords: Integer partitions, Polynomials, Partition inequality

Mathematics Subject Classification: Primary 05A17, 11P82, Secondary 05A20
\end{abstract}

\section{Introduction and main results}

Bessenrodt and Ono [3] discovered an interesting inequality for partition numbers $p(n)$. Their discovery nudged further research and new results in several directions.

A partition $\lambda$ of $n$ is any non-increasing sequence $\lambda_{1}, \ldots, \lambda_{d}$ of positive integers whose sum is $n$. The number of partitions of $n$ is denoted by $p(n)$. Table 1 records the first 10 values and the special case $p(0)=1$.

A partition is called a $k$-colored partition of $n$ if each part can appear in $k$ colors.

Theorem 1.1 ([3]) Let $a$ and $b$ be positive integers. Let $a, b \geq 2$ and $a+b \geq 10$. Then

$$
p(a) p(b)>p(a+b)
$$

The proof depends on results by Rademacher [23] and Lehmer [17] on the size of the partition numbers, built on the circle method by Hardy and Ramanujan. The partition numbers are considered as the coefficients of a weakly modular form, the reciprocal of the Dedekind $\eta$-function [22]. The modularity condition is very strong, and it would be desirable to also have other proofs. author(s) and the source, provide a link to the Creative Commons licence, and indicate if changes were made. The images or other third party material in this article are included in the article's Creative Commons licence, unless indicated otherwise in a credit line to the material. If material is not included in the article's Creative Commons licence and your intended use is not permitted by statutory regulation or exceeds the permitted use, you will need to obtain permission directly from the copyright holder. To view a copy of this licence, visit http://creativecommons.org/licenses/by/4.0/. 
Table 1 Values of $p(n)$ for $0 \leq n \leq 10$

\begin{tabular}{|c|c|c|c|c|c|c|c|c|c|c|c|}
\hline$n$ & 0 & 1 & 2 & 3 & 4 & 5 & 6 & 7 & 8 & 9 & 10 \\
\hline$p(n)$ & 1 & 1 & 2 & 3 & 5 & 7 & 11 & 15 & 22 & 30 & 42 \\
\hline
\end{tabular}

Alanazi et al. [1] came up with an impressive and subtle combinatorial proof. Chern et al. [6] generalized Bessenrodt and Ono's theorem to $k$-colored partitions.

Theorem 1.2 ([6]) Let $a, b, k$ be natural numbers. Let $p_{-k}(n)$ denote the number of $k$ colored partitions of $n$. Let $k>1$. Then

$$
p_{-k}(a) p_{-k}(b)>p_{-k}(a+b)
$$

except for $(a, b, k) \in\{(1,1,2),(1,2,2),(2,1,2),(1,3,2),(3,1,2),(1,1,3)\}$.

Two proofs are given. One depends on the Bessenrodt-Ono inequality and some new ideas, the second is combinatorial and motived by the work of Alanazi et al. [1].

Bessenrodt-Ono type inequalities appeared also in works by Beckwith and Bessenrodt [2] on $k$-regular partitions and Hou and Jagadeesan [15] on the numbers of partitions with ranks in a given residue class modulo 3. Males [18] obtained results for general $t$ and Dawsey and Masri [7] obtained new results for the Andrews spt-function. The authors of this paper generalized the Chern-Fu-Tang Theorem to D'Arcais polynomials, also known as Nekrasov-Okounkov polynomials [6,10,11,14,19,20]. Let

$$
P_{n}(x):=\frac{x}{n} \sum_{k=1}^{n} \sigma(k) P_{n-k}(x)
$$

with $\sigma(k):=\sum_{d \mid k} d$ and the initial condition $P_{0}(x)=1$. Then $p_{-k}(n)=P_{n}(k)$ and $p(n)=p_{-1}(n)=P_{n}(1)$.

Theorem 1.3 ([11]) Let $a, b \in \mathbb{N}, a+b>2$, and $x>2$. Then

$$
P_{a}(x) P_{b}(x)>P_{a+b}(x) .
$$

The case $x=2$ is true for $a+b>4$.

The proof is based on the result for 2-colored partitions [6], Lehmer's [17] lower and upper bound on the partition numbers, and a detailed analysis of the growth of the derivative of $P_{a, b}(x)$.

These results are related to the work by Griffin et al. [9] on Jensen polynomials and their hyperbolicity. This includes work by Nicolas [21] and DeSalvo and Pak [8] on the $\log$-concavity of the partition function $p(n)$ for $n>25$, and results and a conjecture by Chen et al. [5] for the higher order Turán inequalities. Related to their work on the Bessenrodt-Ono inequality, Chern et al. came up with a subtle and explicit conjecture on $k$-colored partitions. The positivity of the discriminant in the case of degree 2 is equal to the log-concavity of the considered sequences coded in the Jensen polynomials.

Conjecture 1 ([6]) Let $n>m \geq 1$ and $k \geq 2$. Then, except for $(k, n, m)=(2,6,4)$,

$$
p_{-k}(n-1) p_{-k}(m+1) \geq p_{-k}(n) p_{-k}(m)
$$


The conjecture was extended to D'Arcais polynomials.

Conjecture 2 ([11]) Let $a>b \geq 0$ be integers. Then for all $x \geq 2$ :

$$
\Delta_{a, b}(x):=P_{a-1}(x) P_{b+1}(x)-P_{a}(x) P_{b}(x) \geq 0,
$$

except for $b=0$ and $(a, b)=(6,4)$. The inequality (1.1) is still true for $x \geq 3$ for $b=0$ and for $x \geq x_{6,4}$ for $(a, b)=(6,4)$. Here $x_{a, b}$ is the largest real root of $\Delta_{a, b}(x)$.

Based on a recently obtained exact formula of Rademacher type (based on the circle method) for $P_{n}(x)$ with $x>0$ and $n>\frac{x}{24}$ obtained by Iskander et al. [16], new strong estimates on the Bessel function, the determination of the main term of $P_{a, b}(x)$, and some sophisticated computer calculation, Bringmann et al. [4] were able to prove the conjecture by Chern et al. They essentially proved the conjecture for $x=2,3,4$ and applied a result by Hoggar on the convolution of log-concave sequences. They further proved that the conjecture by Heim and Neuhauser is true ([4, Corollary 1.3]) for all pairs $(a, b)$, where

$$
b \geq \max \left\{2 x^{11}+\frac{x}{24}, \frac{100}{x-24}+\frac{x}{24}\right\} .
$$

The crux of these methods is that one needs for the general case Rademacher type formulas, and has to check the conjecture for each $x$ for finitely many cases. In the discrete case for the Bessenrodt-Ono inequality there is a combinatorial proof available, this was also requested in [4], see concluding remarks (5), for the Chern-Fu-Tang conjecture.

In this paper we offer a new proof for the Bessenrodt-Ono inequality for partition numbers. Ingredients are the well-known recurrence property:

$$
n p(n)=\sum_{k=1}^{n} \sigma(k) p(n-k),
$$

and an elementary upper bound of $\sigma(n)$ and lower bound of $p(n)$. The proof also perfectly fits to the Bessenrodt-Ono inequality for $k$-colored partitions $p_{-k}(n)$ and D'Arcais polynomials $P_{n}(x)$. With slight modification and including some extra considerations, we obtain new proofs of Theorem 1.2 by Chern-Fu-Tang and Theorem 1.3. Finally, we obtain the following Theorem, to give evidence that the proof method offered in this paper also gives an extension of Theorem 1.3.

Theorem 1.4 Let $a, b \in \mathbb{N}, a+b>4$, and $x>1$.8. Then

$$
P_{a}(x) P_{b}(x)>P_{a+b}(x) .
$$

It is hoped that the results of this paper lead to a new proof of the former Conjecture 1 by Chern-Fu-Tang [4] and to a proof of Conjecture 2 .

\section{New proof of the Bessenrodt-Ono inequality}

We estimate the divisor sum function $\sigma(n)$ and the partition numbers $p(n)$ with the following upper and lower bounds:

$$
\begin{aligned}
& \sigma(n) \leq n(1+\ln (n)), \\
& p(n) \geq \sum_{k=1}^{m} \frac{1}{k !}\left(\begin{array}{l}
n-1 \\
k-1
\end{array}\right) \text { for all } m \in \mathbb{N} .
\end{aligned}
$$


The upper bound for $\sigma(n)$ follows easily by integral comparison. We have a strict upper bound for $n>1$. There are exactly $\left(\begin{array}{c}n-1 \\ k-1\end{array}\right)$ ways to represent $n$ as a sum of exactly $k$ positive integers. Thus, we have at most $k$ ! compositions representing the same partition. For a generalization we refer to Sect. 3 and the relation to associated Laguerre polynomials.

Proof Let $A:=2$ and $B:=10$. Let $n \geq B$. We say the statement $S(n)$ is true if for all partitions $n=a+b$ with $a, b \geq A$ :

$$
p_{a, b}(n):=p(a) p(b)-p(a+b)>0 .
$$

By symmetry the claim can be reduced to all pairs $(a, b)$ with $A \leq b \leq a$. We assume that $n>N_{0}>1$ and $S(m)$ are true for all $B \leq m \leq n-1$. For $B \leq \ell \leq N_{0}$ we show $S(\ell)$ by a direct computer calculation with PARI/GP.

Note, it is sufficient to prove $S(n)$ for fixed $A \leq b \leq a$ with $a+b=n$. We have introduced the constants $A, B$ and $N_{0}$ to make the generalization of the given proof in our applications transparent. It will turn out that we can choose $N_{0}=2184$.

We utilize the recurrence (1.2) and obtain for $p_{a, b}(n)=L+R$ the expressions:

$$
\begin{aligned}
L & :=-\sum_{k=1}^{b} \frac{\sigma(k+a)}{a+b} p(b-k), \\
R & :=\sum_{k=1}^{a}\left(\frac{\sigma(k)}{a} p(a-k) p(b)-\frac{\sigma(k)}{a+b} p(a+b-k)\right) .
\end{aligned}
$$

We show that $p_{a, b}(n)>0$. Further, we will refine the right $\operatorname{sum} R$ into

$$
R=R_{1}+R_{2}+R_{3}
$$

\subsection{Left sum $L$}

We have

$$
L=-\sum_{k=1}^{b} \frac{\sigma(k+a)}{a+b} p(b-k)>-b p(b)(1+\ln (a+b)) .
$$

\subsection{Right sum $R$}

The dominant term is related to $k=1$ appearing in the right sum $R$. Note that the induction hypothesis cannot be applied in general to all terms. Therefore, we decompose the right sum $R$ into three parts. Let

$$
k_{0}:=a-\max \{B-b, A\}+1 .
$$

Thus, $k_{0}=a-\max \{B-b-1, A-1\}=a-\max \{9-b, 1\}$ for $A=2$ and $B=10$. Let

$$
\begin{aligned}
R_{1} & :=\sum_{k=1}^{1} f_{k}(a, b), R_{2}:=\sum_{k=2}^{k_{0}-1} f_{k}(a, b), R_{3}:=\sum_{k=k_{0}}^{a} f_{k}(a, b), \\
f_{k}(a, b) & :=\frac{\sigma(k)}{a} p(a-k) p(b)-\frac{\sigma(k)}{a+b} p(a+b-k) .
\end{aligned}
$$




\subsubsection{The sum $R_{1}$}

The first sum related to $k=1$ is simplified by the induction hypothesis:

$$
-p(a+b-1)>-p(a-1) p(b)
$$

Thus, we obtain the lower bound:

$$
R_{1}=\frac{p(a-1) p(b)}{a}-\frac{p(a+b-1)}{a+b}>\frac{b}{2 a^{2}} p(a-1) p(b) .
$$

\subsubsection{The sum $R_{2}$}

The second sum, using again the induction hypothesis, can be estimated from below with 0 . This will be sufficient for our purpose: $R_{2}>0$.

\subsubsection{The sum $R_{3}$}

We split the third sum again into three parts: $R_{3}=R_{31}+R_{32}+R_{33}$, where

$$
\begin{aligned}
R_{31}:=\sum_{k=k_{0}}^{a-A} f_{k}(a, b) & =\sum_{k=k_{0}}^{a-A} \sigma(k)\left(\frac{p(a-k) p(b)}{a}-\frac{p(a+b-k)}{a+b}\right) \\
& >5(4-p(8))(1+\ln (a)), \\
R_{32}:=\sum_{k=a-A+1}^{a-1} f_{k}(a, b) & =\sigma(a-1)\left(\frac{p(1) p(b)}{a}-\frac{p(b+1)}{a+b}\right) \\
& >-\frac{\sigma(a-1) p(b)}{a}>-p(b)(1+\ln (a)), \\
R_{33} & :=\sum_{k=a}^{a} f_{k}(a, b)
\end{aligned}
$$

We first use that $p(9-b) p(b) \geq p(9)$. This leads to at most 5 summands. The next estimation in (2.6) follows from $p(a-k) \geq p(2)$. For the second estimation we use that

$$
p(b) \geq p(2)=2, \frac{1}{a+b}<\frac{1}{a}, \text { and } p(a+b-k) \leq p(8) .
$$

We also refer to (2.1). The estimation in (2.7) follows from the obvious inequality $\frac{1}{a+b}<\frac{1}{a}$ and $p(b+1)<2 p(b)$. Thus,

$$
R_{3}>-(90+p(b))(1+\ln (a)) .
$$

Since $b \geq 2,90 \leq \frac{45}{2} b p(b), \frac{1}{a+b} \geq \frac{1}{2 a}$, and $a-1<a<a+b$ we obtain

$$
R_{3}>-b p(b)(1+\ln (a+b)) \frac{1+45}{2} \text {. }
$$

\subsection{Final step}

Putting everything together leads to

$$
\begin{aligned}
p_{a, b}(n) & \left.>\frac{b p(b)}{2 a^{2}}\left(-48 a^{2}(1+\ln (a+b))+p(a-1)\right)\right) \\
& >\frac{b p(b)}{2 a^{2}}\left(-48 a^{2}(1+\ln (2 a))+\sum_{\ell=1}^{5} \frac{1}{\ell !}\left(\begin{array}{l}
a-2 \\
\ell-1
\end{array}\right)\right) .
\end{aligned}
$$


In the last step we used the property (2.2). For $a \rightarrow \infty$ we can immediately observe that this is positive since the sum is a polynomial of degree 4 in $a$ which grows faster than $48 a^{2}(1+\ln (2 a))$. In fact the expression (2.8) is positive for all $a \geq 1093$. Note that if $a<1093$ then $a+b \leq 2 a \leq 2184=N_{0}$. Therefore, we have shown that $p_{a, b}(n)>0$, which proves the theorem.

\section{Applications}

We extend the proof method presented in Sect. 2 to prove the Bessenrodt-Ono inequality for $k$-colored partitions and its extension to D'Arcais polynomials. Let $k \in \mathbb{N}$. Then $P_{n}(k)$ is equal to the $k$-colored partition number and $p(n)=P_{n}(1)$. We define

$$
P_{a, b}(x):=P_{a}(x) P_{b}(x)-P_{a+b}(x)
$$

Before we start, we fix the following lower bound for the D'Arcais polynomials $P_{n}(x)$. Let $x$ and $\alpha$ be real numbers with $x \geq 0$ and $\alpha>-1$. Let $L_{n}^{(\alpha)}(x)$ be the $\alpha$-associated Laguerre polynomial. Then $P_{n}(x) \geq \frac{x}{n} L_{n-1}^{(1)}(-x)$. We refer to [13]. This implies

$$
P_{n}(x) \geq \sum_{k=1}^{m}\left(\begin{array}{l}
n-1 \\
k-1
\end{array}\right) \frac{x^{k}}{k !} \text { for all } m \in \mathbb{N} .
$$

\subsection{Bessenrodt-Ono for $x>3$ and arbitrary $a$ and $b$}

We first prove that $P_{a, b}(x)>0$ for $x>3$ is true for all $a$ and $b \in \mathbb{N}$. Since there are no restrictions on $a$ and $b$, the proof will be straightforward.

Proposition 3.1 Let $a$ and $b$ be positive integers with $a, b \geq 1$ and $x$ a real number with $x>3$. Then $P_{a, b}(x)>0$.

Proof We follow the proof by induction of the Bessenrodt-Ono inequality presented in Sect. 2. Let $n \geq 2$. The statement $S(n)$ is true if for all partitions $n=a+b$ with $a, b \geq 1$ holds $P_{a, b}(x)>0$ for $x>3$. Let $1 \leq b \leq a$. Let $n>N_{0}$ and $S(m)$ be true for all $2 \leq m \leq n-1$. We show that it is sufficient to put $N_{0}=16$. Note that $S(n)$ is true for all $2 \leq a+b \leq N_{0}$ (see Table 3). Let $P_{a, b}(x)=L+R$ with $L$ and $R$ defined as in (2.3) and (2.4), where we have to replace $p(n)$ by $P_{n}(x)$.

\subsubsection{Left sum $L$}

The left sum satisfies

$$
L>-b P_{b}(x)(1+\ln (a+b)) .
$$

\subsubsection{Right sum $R$}

We take care about the dominating term for $k=1$. Similar to (2.5) with

$$
f_{k}(a, b):=\frac{\sigma(k)}{a} P_{a-k}(x) P_{b}(x)-\frac{\sigma(k)}{a+b} P_{a+b-k}(x)
$$

we study $R=R_{1}+R_{2}+R_{3}$. By the induction hypothesis we get $R_{2}>0$. And since we have no extra condition on $a$ and $b$ we also get $R_{3} \geq 0$. Thus, only $R_{1}$ attached to $k=1$ 
contributes and leads to

$$
R>\frac{b}{2 a^{2}} P_{a-1}(x) P_{b}(x)
$$

\subsubsection{Final step}

Putting everything together leads to

$$
\begin{aligned}
P_{a, b}(x) & >\frac{b P_{b}(x)}{2 a^{2}}\left(-2 a^{2}(1+\ln (a+b))+P_{a-1}(x)\right) \\
& >\frac{b P_{b}(x)}{2 a^{2}}\left(-2 a^{2}(1+\ln (2 a))+\sum_{\ell=1}^{6}\left(\begin{array}{l}
a-2 \\
\ell-1
\end{array}\right) \frac{3^{\ell}}{\ell !}\right) .
\end{aligned}
$$

In the last step we used the property (3.1) and that $x>3$. We obtain that the expression (3.3) is positive for all $a \geq 12$. Now we can check that (3.2) is positive already for $a \in$ $\{9,10,11\}$. Since the leading coefficient $\frac{1}{a ! b !}-\frac{1}{(a+b) !}$ of $P_{a, b}(x)$ is positive, we only have to check the largest real zero of all remaining $P_{a, b}(x)$. This was done for $2 \leq b+a \leq N_{0}=16$ with PARI/GP (Table 3). Note that in the case of $P_{1,1}(x)=(x-3) \frac{x}{2}$ the largest real zero is exactly 3 .

\subsection{2-colored partitions}

Chern-Fu-Tang ([6], Theorem 1.2) proved the following result.

Theorem 3.2 Let $a$ and $b$ be positive integers with $a, b \geq 1$ and $n=a+b \geq 5$. Then $P_{a, b}(2)>0$.

Proof We have $A=1, B=5$, and $k_{0}=a-\max \{5-b, 1\}+1$. Let $n \geq 5$ and $S(n)$ be the statement: $P_{a, b}(2)>0$ for all $a, b \geq 1$ with $n=a+b$. A numerical calculation with PARI/GP shows that $S(m)$ is true for all $5 \leq m \leq N_{0}=54$. We prove $S(n)$ by induction on $n$. Let $n=a+b>N_{0}$ and $1 \leq b \leq a$. Let $P_{a, b}(2)=L+R$. Then

$$
L>-b P_{b}(2)(1+\ln (a+b)) \text {. }
$$

Further,

$$
R_{1}>\frac{b}{2 a^{2}} P_{a-1}(2) P_{b}(2) .
$$

We have $R_{2} \geq 0$ by the induction hypothesis and $R_{3} \geq 0$ for $b>3$. Moreover

$$
\frac{P_{a-k}(2) P_{b}(2)}{a}-\frac{P_{a+b-k}(2)}{a+b}>\frac{P_{a-k}(2) P_{b}(2)-P_{a+b-k}(2)}{a} .
$$

From the induction hypothesis and Table 2 we see that this is non-negative for $a-k \geq 2$ or $b \geq 2$. For $a-k=1=b$ we obtain $\left(P_{1}(2)\right)^{2}-P_{2}(2)=-1$. This leads to

$$
\begin{aligned}
P_{a, b}(2) & >\frac{b P_{b}(2)}{2 a^{2}}\left(-3 a^{2}(1+\ln (a+b))+P_{a-1}(2)\right) \\
& >\frac{b P_{b}(2)}{2 a^{2}}\left(-3 a^{2}(1+\ln (2 a))+\sum_{\ell=1}^{6} \frac{2^{\ell}}{\ell !}\left(\begin{array}{l}
a-2 \\
\ell-1
\end{array}\right)\right) .
\end{aligned}
$$

In the last step we used the property (3.1). For $a \rightarrow \infty$ we can immediately observe that this is positive since the sum is a polynomial of degree 5 in $a$, which grows faster than $a^{2}(1+\ln (2 a))$. In fact, the expression (3.4) is positive for all $a \geq 28$. For the remaining $5 \leq a+b \leq 54$ we have checked with PARI/GP that $P_{a, b}(2)>0$. 
Table 2 Values of $P_{a, b}(2)$ for $a, b \in\{1,2,3,4\}$

\begin{tabular}{lllll}
\hline$a \backslash b$ & 1 & 2 & 3 & 4 \\
\hline 1 & -1 & 0 & 0 & 4 \\
2 & 0 & 5 & 14 & 35 \\
3 & 0 & 14 & 35 & 90 \\
4 & 4 & 35 & 90 & 215 \\
\hline
\end{tabular}

\subsection{Proof of Theorem 1.4}

Let $n \geq 5$. The statement $S(n)$ is true if for all partitions $n=a+b$ with $a, b \geq 1$ holds $P_{a, b}(x)>0$ for $x>1.8$. Let $1 \leq b \leq a$. Let $n>N_{0}$ and $S(m)$ be true for all $5 \leq m \leq n-1$. We show that it is sufficient to put $N_{0}=36$. Note that $S(n)$ is true for all $1 \leq a+b \leq 36$ (compare Table 3). Let $P_{a, b}(x)=L+R$ with $L$ and $R$ defined as in (2.3) and (2.4), where we have to replace $p(n)$ by $P_{n}(x)$

\subsubsection{Left sum $L$}

The left sum satisfies

$$
L>-b P_{b}(x)(1+\ln (a+b)) .
$$

\subsubsection{Right sum $R$}

We study $R=R_{1}+R_{2}+R_{3}$. By the induction hypothesis we get $R_{2}>0 . R_{1}$ associated to $k=1$ leads to

$$
R_{1}>\frac{b}{2 a^{2}} P_{a-1}(x) P_{b}(x)
$$

We find that $R_{32}=0$ and $R_{33} \geq 0$. For $b \geq 4$ we can apply the induction hypothesis and obtain $R_{31}>0$. In case $1 \leq b \leq 3$ we find from Table 3 that $P_{a-k, b}(1.8)>0$ for $(a-k, b) \notin\{(1,1),(1,2),(1,3),(2,1),(3,1)\}$.

It can be checked that the polynomials $P_{1,1}(x)=\frac{1}{2} x^{2}-\frac{3}{2} x, P_{2,1}(x)=\frac{1}{3} x^{3}-\frac{4}{3} x$ and $P_{3,1}(x)=\frac{1}{8} x^{4}+\frac{3}{4} x^{3}-\frac{9}{8} x^{2}-\frac{7}{4} x$ are monotonically increasing for $x \geq 1.8$. Thus,

$$
R_{31}>-1.5648(1+\ln (a))
$$

\subsubsection{Final step}

Putting everything together leads to

$$
\begin{aligned}
P_{a, b}(x) & >\frac{b P_{b}(x)}{2 a^{2}}\left(-3.8 a^{2}(1+\ln (a+b))+P_{a-1}(x)\right) \\
& >\frac{b P_{b}(x)}{2 a^{2}}\left(-3.8 a^{2}(1+\ln (2 a))+\sum_{\ell=1}^{9}\left(\begin{array}{l}
a-2 \\
\ell-1
\end{array}\right) \frac{1.8^{\ell}}{\ell !}\right) .
\end{aligned}
$$

In the last step we used the property (3.1) and that $x>1.8$. We obtain that the expression (3.6) is positive for all $a \geq 30$. Now we can check that (3.5) is already positive for $a \in\{19,20, \ldots, 29\}$. Since the leading coefficient of $P_{a, b}(x)$ is positive we only have to determine the largest real zero of all remaining $P_{a, b}(x)$. This was done for $1 \leq b+a \leq 36$ with PARI/GP (compare Table 3). 
Table 3 Approximative largest real zeros of $P_{a, b}(x)$ for $1 \leq a \leq 16,1 \leq b \leq 15$

\begin{tabular}{llllllllllllllll}
\hline$a \backslash b$ & 1 & 2 & 3 & 4 & 5 & 6 & 7 & 8 & 9 & 10 & 11 & 12 & 13 & 14 & 15 \\
\hline 1 & 3.0 & 2.0 & 2.0 & 1.7 & 1.7 & 1.6 & 1.6 & 1.5 & 1.5 & 1.4 & 1.5 & 1.4 & 1.4 & 1.4 & 1.4 \\
2 & 2.0 & 1.4 & 1.2 & 1.1 & 1.1 & 1.0 & 1.0 & 0.9 & 0.9 & 0.9 & 0.9 & 0.9 & 0.9 & 0.8 & 0.8 \\
3 & 2.0 & 1.2 & 1.2 & 1.0 & 1.0 & 0.9 & 0.9 & 0.8 & 0.9 & 0.8 & 0.8 & 0.8 & 0.8 & 0.8 & 0.8 \\
4 & 1.7 & 1.1 & 1.0 & 0.9 & 0.9 & 0.8 & 0.8 & 0.7 & 0.7 & 0.7 & 0.7 & 0.6 & 0.6 & 0.6 & 0.6 \\
5 & 1.7 & 1.1 & 1.0 & 0.9 & 0.9 & 0.7 & 0.8 & 0.7 & 0.7 & 0.7 & 0.7 & 0.6 & 0.7 & 0.6 & 0.6 \\
6 & 1.6 & 1.0 & 0.9 & 0.8 & 0.7 & 0.7 & 0.7 & 0.6 & 0.6 & 0.6 & 0.6 & 0.5 & 0.5 & 0.5 & 0.5 \\
7 & 1.6 & 1.0 & 0.9 & 0.8 & 0.8 & 0.7 & 0.7 & 0.6 & 0.6 & 0.6 & 0.6 & 0.5 & 0.6 & 0.5 & 0.5 \\
8 & 1.5 & 0.9 & 0.8 & 0.7 & 0.7 & 0.6 & 0.6 & 0.6 & 0.6 & 0.5 & 0.5 & 0.5 & 0.5 & 0.5 & 0.5 \\
9 & 1.5 & 0.9 & 0.9 & 0.7 & 0.7 & 0.6 & 0.6 & 0.6 & 0.6 & 0.5 & 0.5 & 0.5 & 0.5 & 0.5 & 0.5 \\
10 & 1.4 & 0.9 & 0.8 & 0.7 & 0.7 & 0.6 & 0.6 & 0.5 & 0.5 & 0.5 & 0.5 & 0.5 & 0.5 & 0.4 & 0.4 \\
11 & 1.5 & 0.9 & 0.8 & 0.7 & 0.7 & 0.6 & 0.6 & 0.5 & 0.5 & 0.5 & 0.5 & 0.5 & 0.5 & 0.4 & 0.4 \\
12 & 1.4 & 0.9 & 0.8 & 0.6 & 0.6 & 0.5 & 0.5 & 0.5 & 0.5 & 0.5 & 0.5 & 0.4 & 0.4 & 0.4 & 0.4 \\
13 & 1.4 & 0.9 & 0.8 & 0.6 & 0.7 & 0.5 & 0.6 & 0.5 & 0.5 & 0.5 & 0.5 & 0.4 & 0.4 & 0.4 & 0.4 \\
14 & 1.4 & 0.8 & 0.8 & 0.6 & 0.6 & 0.5 & 0.5 & 0.5 & 0.5 & 0.4 & 0.4 & 0.4 & 0.4 & 0.4 & 0.4 \\
15 & 1.4 & 0.8 & 0.8 & 0.6 & 0.6 & 0.5 & 0.5 & 0.5 & 0.5 & 0.4 & 0.4 & 0.4 & 0.4 & 0.4 & 0.4 \\
16 & 1.4 & 0.8 & 0.7 & 0.6 & 0.6 & 0.5 & 0.5 & 0.5 & 0.5 & 0.4 & 0.4 & 0.4 & 0.4 & 0.4 & 0.4 \\
\hline
\end{tabular}

Funding Open Access funding enabled and organized by Projekt DEAL.

\section{Author details}

${ }^{1}$ Lehrstuhl A für Mathematik, RWTH Aachen University, 52056 Aachen, Germany, ${ }^{2}$ Kutaisi International University, 5/7, Youth Avenue, 4600 Kutaisi, Georgia.

Received: 14 July 2021 Accepted: 29 October 2021 Published online: 26 November 2021

\section{References}

1. Alanazi, A.A., Gagola, S.M., III., Munagi, A.O.: Combinatorial proof of a partition inequality of Bessenrodt-Ono. Ann. Comb. 21 (3), 331-337 (2017)

2. Beckwith, O., Bessenrodt, C.: Multiplicative properties of the number of k-regular partitions. Ann. Comb. 20(2), 231-250 (2016)

3. Bessenrodt, C., Ono, K.: Maximal multiplicative properties of partitions. Ann. Comb. 20(1), 59-64 (2016)

4. Bringmann, K., Kane, B., Rolen, L., Tripp, Z.: Fractional partitions and conjectures of Chern-Fu-Tang and HeimNeuhauser. Trans. Am. Math. Soc. Ser. B 8(21), 615-634 (2021)

5. Chen, W., Jia, D., Wang, L.: Higher order Turán inequalities for the partition function. Trans. Am. Math. Soc. 372 2143-2165 (2019)

6. Chern, S., Fu, S., Tang, D.: Some inequalities for k-colored partition functions. Ramanujan J. 46, 713-725 (2018)

7. Dawsey, M.L., Masri, R.: Effective bounds for the Andrews spt-function. Forum Math. 31(3), 743-767 (2019)

8. DeSalvo, S., Pak, I.: Log-concavity of the partition function. Ramanujan J. 38, 61-73 (2015)

9. Griffin, M., Ono, K., Rolen, L., Zagier, D.: Jensen polynomials for the Riemann zeta function and other sequences. Proc. Natl. Acad. Sci. USA 116(23), 11103-11110 (2019)

10. Han, G.: The Nekrasov-Okounkov hook length formula: refinement, elementary proof and applications. Ann. Inst. Fourier (Grenoble) 60(1), 1-29 (2010)

11. Heim, B., Neuhauser, M.: The Dedekind eta function and D'Arcais-type polynomials. Res. Math. Sci. 7, 3 (2020)

12. Heim, B., Neuhauser, M.: Polynomization of the Chern-Fu-Tang conjecture. Res. Numb. Theory 7, 26 (2021)

13. Heim, B., Luca, F., Neuhauser, M.: Recurrence relations for polynomials obtained by arithmetic functions. Int. J. Numb. Theory 15(6), 1291-1303 (2019)

14. Heim, B., Neuhauser, M., Tröger, R.: Polynomization of the Bessenrodt-Ono inequality. Ann. Comb. 24(4), 697-709 (2020)

15. Hou, E., Jagadeesan, M.: Dyson's partition ranks and their multiplicative extension. Ramanujan J. 45(3), 817-839 (2018)

16. Iskander, J., Jain, V., Talvola, V.: Exact formulae for the fractional partition functions. Res. Numb. Theory 6(3), 1-17 (2020)

17. Lehmer, D.H.: On the remainders and convergence of the series for the partition functions. Trans. Am. Math. Soc. 46 362-373 (1939)

18. Males, J.: Asymptotic equidistribution and convexity for partition ranks. Ramanujan J. 54(2), 397-413 (2021)

19. Nekrasov, N., Okounkov, A.: Seiberg-Witten theory and random partitions. In: P. Etingof, V. Retakh, I. M. Singer (eds.) The unity of mathematics. In honor of the ninetieth birthday of I. M. Gelfand. Papers from the conference held in Cambridge, MA, USA, August 31-September 4, 2003. Progr. Math., vol. 244, Birkhäuser Boston, pp. 525-596 (2006)

20. Newman, M.: An identity for the coefficients of certain modular forms. J. Lond. Math. Soc. 30, 488-493 (1955) 
21. Nicolas, J.-L.: Sur les entiers $N$ pour lesquels il y a beaucoup des groupes abéliens d'ordre N. Ann. Inst. Fourier $\mathbf{2 8 ( 4 ) , ~}$ $1-16(1978)$

22. Ono, K.: The Web of Modularity: Arithmetic of the Coefficients of Modular Forms and q-series. CBMS Regional Conference Series in Mathematics, vol. 102. American Mathematical Society, Providence (2004)

23. Rademacher, H.: A convergent series for the partition function $p(n)$. Proc. Natl. Acad. Sci. USA 23, 78-84 (1937)

\section{Publisher's Note}

Springer Nature remains neutral with regard to jurisdictional claims in published maps and institutional affiliations. 